\title{
Non-Radial Oscillations in an Axisymmetric MHD Incompressible Fluid
}

\author{
A. Satya Narayanan, Indian Institute of Astrophysics, Bangalore 560 034, India. \\ e-mail: satya@iiap.ernet.in
}

\begin{abstract}
It is well known from Helioseismology that the Sun exhibits oscillations on a global scale, most of which are non-radial in nature. These oscillations help us to get a clear picture of the internal structure of the Sun as has been demonstrated by the theoretical and observational (such as GONG) studies. In this study we formulate the linearised equations of motion for non-radial oscillations by perturbing the MHD equilibrium solution for an axisymmetric incompressible fluid. The fluid motion and the magnetic field are expressed as scalars $U, V, P$ and $T$, respectively. In deriving the exact solution for the equilibrium state, we neglect the contribution due to meridional circulation. The perturbed quantities $U_{*}, V_{*}, P_{*}, T_{*}$ are written in terms of orthogonal polynomials. A special case of the above formulation and its stability is discussed.
\end{abstract}

Key words. Non-radial oscillations-MHD-axisymmetry.

\section{Introduction}

Studies of stellar pulsations, in particular the Sun, is interesting both from the theoretical and observational point of view. Stars such as the Cepheids are known to pulsate with large amplitudes. In the case of the Sun, several thousand individual modes have been identified. With careful observation, frequencies for as many as $10^{6}$ modes can be determined accurately. Helioseismology deals with the study of the interior of the Sun from the observed frequencies of modes of oscillations. It is interesting to note that most of the modes are non-radial in nature. For more details about non-radial oscillations, refer to Unno et al. (1989), Christensen-Dalsgard (1997).

In order to study oscillations, it is important to determine the equilibrium state of stars which are under the combined action of gravitational, thermal and magnetic forces. In most of the studies related to Helioseismology, the equilibrium state is taken to be hydrostatic, where the pressure gradients are balanced by gravity in the absence of fluid motions. However, stars like the Sun have magnetic field both inside as well as on the outer atmospheres. It would be interesting to study the equilibrium state with magnetic field and motions included, before venturing into the study of oscillations. There have been several studies on the equilibrium of stars, in particular the Sun by Chandrasekhar (1956), Ferraro (1954), Pendergast (1956), Nakagawa \& Trehan (1968), Gokhale \& Hiremath (1993), Satya Narayanan (1996), Del Zanna \& Chiuderi (1996), Neukirch \& Tastatter (1999) who have investigated the combined effects of fluid motions and magnetic fields. 


\section{Equilibrium solution}

In a cylindrical coordinate system $(y, \phi, z)$, the hydromagnetic equations for equilibrium in an incompressible medium with infinite electrical conductivity in which axial symmetry prevails can be written in the form of the following system of coupled partial differential equations for the scalars $P, T, U, V$ which define the magnetic and velocity fields (Satya Narayanan 1996).

$$
\begin{gathered}
{\left[y^{2} U, y^{2} P\right]=0, \quad\left[y^{2} U, T\right]+\left[V, y^{2} P\right]=0,} \\
{\left[y^{2} T, y^{2} P\right]+\left[y^{2} U, y^{2} V\right]=0,} \\
{\left[\triangle_{5} P, y^{2} P\right]-\left[\triangle_{5} U, y^{2} U\right]+y \frac{\partial}{\partial y}\left\{T^{2}-V^{2}\right\}=0 .}
\end{gathered}
$$

Here, $[X, Y]=\partial X / \partial y \partial Y \partial z-\partial X / \partial z \partial Y \partial y$. In stars like the Sun, the meridional motion $U$ is negligible. Neglecting $U$, equations (1)-(3) can be simplified to yield

$$
\triangle_{5} P+\frac{1}{y^{2}} G\left(y^{2} P\right)+y^{2} g\left(y^{2} P\right)=\Phi\left(y^{2} P\right) .
$$

$G, g$ and $\Phi$ are arbitrary functions of $y^{2} P$. Equation (4) represents the general integral of the equilibrium solution for the case $U=0$. The above equation is highly nonlinear. However, for certain specific choices of $G, g$ and $\Phi$, the equation can be made linear.

The linear equation is

$$
\triangle_{5} P+\alpha^{2} P=k-\beta y^{2} / 2
$$

where $\alpha, \beta$ and $k$ are constants.

The solution in spherical polars (by a simple transformation) is

$$
P=\sum_{n=0}^{\infty} A_{n} \frac{J_{n+3 / 2}(\alpha r)}{(\alpha r)^{3 / 2}} C_{n}^{3 / 2}(\mu)+\frac{k}{\alpha^{2}}+\frac{4 \beta}{\alpha^{4}}-\frac{\beta}{2 \alpha^{2}} r^{2}\left(1-\mu^{2}\right)
$$

$\mu=r \cos \theta$. The boundary condition is similar to that discussed by Hiremath \& Gokhale (1995).

\section{Oscillations}

In order to study the oscillations, we perturb the scalars $U, V, P, T$ as follows

$$
U=U_{0}+\delta U, V=V_{0}+\delta V, P=P_{0}+\delta P, T=T_{0}+\delta T .
$$

The linearised equations for the oscillations can be written as

$$
\begin{gathered}
\frac{\partial \delta P}{\partial t}=\left[y^{2} \delta U, y^{2} P_{0}\right] \\
\frac{\partial \delta T}{\partial t}=\left[V_{0}, y^{2} \delta P\right]+\left[\delta V, y^{2} P_{0}\right]-\left[T_{0}, y^{2} \delta U\right]
\end{gathered}
$$




$$
\begin{gathered}
y^{3} \frac{\partial \delta V}{\partial t}=\left[T_{0}, y^{2} \delta P\right]+\left[y^{2} \delta T, y^{2} P_{0}\right]-\left[y^{2} V_{0}, y^{2} \delta U\right] \\
y \triangle_{5} \frac{\partial \delta U}{\partial t}-\left[\triangle_{5} P_{0}, y^{2} \delta P\right]-\left[\triangle_{5} \delta P, y^{2} P_{0}\right]=2 y \frac{\partial}{\partial z}\left(T_{0} \delta T-V_{0} \delta V\right) .
\end{gathered}
$$

$\delta U, \delta V, \delta P, \delta T$ denote perturbations while $V_{0}, P_{0}, T_{0}$ denote the basic state. $U_{0}$ has been neglected.

The basic equations which are derived in the cylindrical coordinate system can be recast in the spherical polars because of the spherical symmetry. The perturbed quantities are written in terms of spherical harmonics

$$
f(r, \theta, \phi, z)=\sum_{l, m} f_{r}(r) Y_{l}^{m}(\theta, \phi) \exp (-i \omega t)
$$

If we plug in the above equations in the linearised equations of motion, the resulting equations will be quite complicated as the number of terms in each of the expansion is rather large and one will end up in closure problem. However, for the sake of simplicity, we shall restrict our analysis to only the first few terms of the expansion (say $l, m=7$ ). The resulting equations are still complicated and will have to be solved numerically. This is in progress and will be reported subsequently.

An interesting stationary solution of the MHD equations is given by

$$
V=\frac{B}{(4 \pi \rho)^{1 / 2}} \quad \text { and } \quad \frac{p}{\rho}+\frac{B^{2}}{8 \pi \rho}=\text { const. }
$$

The linearised equations of motion can be simplified to yield a single equation for the poloidal field as

$$
\frac{\mathrm{d}^{2} P}{\mathrm{~d} y^{2}}+\frac{1}{y} \frac{\mathrm{d} P}{\mathrm{~d} y}+\left\{k^{2} \chi^{2}-\frac{m^{2}}{y^{2}}\right\} P=0
$$

where $\chi^{2}=1 / p^{2}\left(K+\sigma / 2 V_{A}\right)^{2}-1$. The solution of the above equation is given by $P=A J_{m}(k x y)$ where $J_{m}(x)$ is the Bessel function of order $\mathrm{m}$ and $\mathrm{A}$ is an arbitrary constant. The boundary condition can be shown to be $P(y=R)=0$. The frequency of oscillation of the system can be shown to be $\sigma / 2 V_{A}= \pm 1 / p\left(1+q^{2}\right)^{1 / 2}-\{k+m / p\}$, where $q^{2}=j_{m, n}^{2} /(k R)^{2}$ and $j_{m, n}$ is the nth root of $J_{m}(x)=0$. It is easy to see that the frequency of oscillation $\sigma$ is always real and hence the system is stable to small perturbations.

\section{Conclusions}

An exact solution of the MHD equilibrium with the assumption of axial symmetry and large conductivity has been presented. The meridional circulation has been neglected. We have formulated the linearised equations of motion for oscillations about the equilibrium solution. We retain only a few terms in the expansion. The resulting equations will be solved later. Finally, a specific steady solution and its stability has been discussed. 


\section{References}

Chandrasekhar, S. 1956, Astrophys. J., 124, 232.

Christensen-Dalsgard, J. 1997, Lecture Notes on Stellar Oscillations.

Del Zanna, L., Chiuderi, C. 1996, Astr. Astrophys., 310, 341.

Ferraro, V. C. A. 1954 Astrophys. J., 119, 407.

Gokhale, M. H., Hiremath, K. M., 1993, Astrophys. J., 407, 350.

Hiremath, K. M., Gokhale, M. H. 1995, Astrophys. J., 448, 437.

Nakagawa, Y., Trehan, S. K. 1968, Astrophys. J., 151, 1111.

Neukirch, T., Rastatter, L. 1999, Astron. Astrophys, (in press).

Pendergast, K. H. 1956, Astrophys. J., 123, 498.

Satya Narayanan, A. 1996, Physica Scripta, 53, 638.

Unno, W., Osaki, Y., Ando, H., Shibahashi, H. 1989, In Nonradial Oscillations of Stars, (University of Tokyo Press). 